\title{
Structure and dynamics of near-threshold leptons driven by dipolar interactions: an accurate computational study for the DNA purinic bases ${ }^{\star, \star \star}$
}

Fabio Carelli and Francesco Antonio Gianturco ${ }^{\mathrm{a}}$

Institut für Ionen Physik und Angewandte Physik, Universität Innsbruck, Technikerstraße 25, 6020 Innsbruck, Austria

Received 16 February 2016 / Received in final form 12 April 2016

Published online 28 June 2016

(c) The Author(s) 2016. This article is published with open access at Springerlink.com

\begin{abstract}
The interaction of low-energy scattering electrons/positrons with molecular targets characterized by a "supercritical" permanent dipole moment $(\gtrsim 2.0 \mathrm{D})$ presents special physical characteristics that affect their spatial distributions, around the nuclear network of the molecular partners, both above and below the energy thresholds. Such special states are described as either dipole scattering states (DSS) above thresholds or as dipole bound states (DBS) below thresholds. The details of their respective behaviour will be presented and discussed in this work in the case of the purinic DNA bases of adenine and guanine. The behavior of the additional electron, in particular, will be discussed in detail by providing new computational results that will be related to the findings from recent experiments on the same DNA bases, confirming the transient electron's behaviour surmised by them.
\end{abstract}

\section{Introduction}

An interesting finding by Fermi and Teller [1,2], which was rediscovered once again in the 60 's $[3,4]$, dealt with the special properties of a stationary electric dipole of supporting bound states only if the dipole moment exceeds a critical value of about 0.639 a.u. They also discovered that the same critical value holds for both a physical dipole and a point dipole, where the anisotropic potential acquires the simple form:

$$
V(r, \theta)=\frac{1}{4 \pi \epsilon_{0}} \frac{\mu \cos \theta}{r^{2}}
$$

with $\vec{\mu}=q \vec{r}[5]$.

The above critical value of $|\vec{\mu}|$ turns out to also be valid for the physical problem in a full $3 \mathrm{D}$ space, while it was recently pointed out that, in the apparently simpler cases in $1 \mathrm{D}$ and $2 \mathrm{D}$, the dipole in question always supports at least one bound state, no matter how small the dipole moment is $[5,6]$.

\footnotetext{
* This work is affectionately dedicated to Michael Allan on the occasion of his official retirement. We wish to this dear friend and outstanding scientist many years to come in the happy pursuit of his many scientific interests.

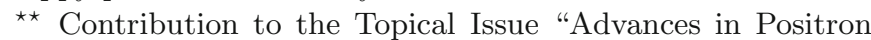
and Electron Scattering", edited by Paulo Limao-Vieira, Gustavo Garcia, E. Krishnakumar, James Sullivan, Hajime Tanuma and Zoran Petrovic.

${ }^{a}$ e-mail: francesco.gianturco@uibk.ac.at
}

The earliest computational studies were usually structural analysis made in order to find out whether or not these additional electrons can be bound via the action of their "supercritical" dipoles [7,8]. Extensive experimental studies on smaller polar molecules have also been carried out over the years [9].

In particular, studies of both experimental and computational nature have looked at molecular systems in a biological context, e.g. the four DNA bases and their close analogues. The presence of free electrons in their environment has been considered important since they are known to be produced via radiative ionization of the surrounding water molecules $[10,11]$ and therefore capable of driving further changes in the biological species interacting with them [12].

Since such molecular systems not only exhibit in the gas-phase large permanent dipoles but often also have large and positive electron affinity (EA) values [8] indicated that they can in principle form two different types of bound molecular anions (radical states for closed shell targets): one with a diffuse extra electron trapped by the dipole potential of equation (1) to the molecular target, and another more strongly bound with the extra electron closer to the molecular nuclear framework. The former is classified as a weakly-bound dipole-bound-state (DBS), while the latter is associated with an excess electron in an unoccupied valence state of the neutral, classified as a valence-bound-state (VBS) [13,14].

Because of the current interest on the behavior of lowenergy positrons, the electron's antiparticle, when made 
to interact at low energies with a large variety of molecular systems, a great deal of studies have also been conducted on finding out their shapes and sizes in cases when they can get weakly bound to molecular biosystems: given the nature of their differences in the interaction forces, the positronic leptons tend to mainly form DBS states, as we shall further discuss below (for additional details see Ref. [15]). Realistic excess electron's calculations in more recent years [16] have estimated that the critical dipole which could give rise to DBS anions is likely to be around 2.5 D, as further confirmed by a variety of experiments [17]. The calculations showed that the excess electron is largely distributed outside of the molecular framework and is much more diffuse than a conventional valence electron's molecular orbital [16].

In the following we shall show that near-threshold scattering states of the external electrons, which shall be termed the dipole-scattering-state (DSS) configurations, bear specific similarities with the weakly-bound DBS states discussed earlier and are located around the target molecules in similar spatial regions, linked to the directions of the permanent super-critical dipoles of the chosen targets. In a series of recent experiments [18], which we shall further discuss below, such near-threshold continuum states are suggested to provide possible doorways to bound anionic species. They could therefore produce an alternative channel to the more disruptive Dissociativeelectron-attachment (DEA) mechanisms often discussed for DNA-related molecular systems (e.g. see Ref. [19]).

In the next section we will briefly review what has been specifically found on the DNA bases about the molecular DBS anions and on their links to the VBS configurations in the same molecules. Section 3 will then "move above the energy threshold" and shall report our quantum scattering methods employed to obtain the dipole-driven scattering states in DNA's molecular systems. Section 4 will present our results for the DSS states of an extra electron for the purinic DNA bases and link them to what has been found in the literature on the corresponding DBS states. Section 5 will draw some general conclusions from the present discussion.

\section{DB and VB anions in DNA bases: a brief discussion}

The fact that polar molecules with a permanent, "supercritical" dipole can form two different types of bound anionic states (usually in the shape of radical anions with enhanced reactivity) has been already known from experiments for a few years: polar species like acetoniltrile $\left(\mathrm{CH}_{3} \mathrm{CN}\right)[16,20]$, the water dimer [21], the nucleobases uracil and thymine [22], and various smaller species [17], have in fact been observed as forming diffuse, weakly bound DBS anions in their ground electronic states. One should also note [9] the earlier extensive review on experiments and modeling of smaller gas-phase molecules, reporting data on the various systems studied in the laboratory of Michael Allan.
The use of Rydberg electron transfer (RET) collisions with rare-gas atoms has been employed to observe extra electron's diffuse bound states on the target polar molecules $[23,24]$. The lifetimes of such weakly-bound DBS anions have been observed in the above type of mesurements to exceed microseconds $[25,26]$. It is also interesting to note at this point that various experiments have reported the evolution of the initially formed DBS anions into more strongly bound VBS anions where the excess electron now can occupy one of virtual molecular orbitals (MO's) of the neutral molecule. Specific experiments discussed the effect of different tautomeric structures, as in uracil [27] or adenine [28] of modifying the initial (weakly bound) DBS states into more strongly bound near-VBS configurations, thereby linking the stabilization of specific configurations of the relevant nucleic bases (NBs) to external perturbations (e.g. water solvent molecules) which can drive the formation of more strongly bound anions in solution, where such NBs are commonly found.

The DBS stabilization via the presence of external solvent molecules has also been discussed in experiments on DNA's NB with a "supercritical" dipole [29]. It was shown that systems with a slightly negative electron affinity as isolated species could develop into more stably bound anionic configurations as the number of clustering solvent molecules is increased, thereby undergoing transitions from a $\sigma^{*}$-type of DBS anion into a more strongly bound $\pi^{*}$-type anion with VBS characteristics [17].

Extensive computational studies on the structural features of the bound $\left[\mathrm{e}^{-}-\mathrm{M}\right]$ complexes have also been carried out on the NB systems by Adamowicz and coworkers (for a rewiew see Ref. [30])), where it was shown that the presence of external molecules to the neutral NB would favour stabilization of DBS configurations of its anion and the subsequent formation of a $\pi^{*}$-type covalent VBS anion.

The "gateway" role for the initially formed DBS anions has also been recently observed in dynamical experiments by Neumark and coworkers $[31,32]$ who investigated binary complexes of iodine-NB systems. It was suggested from their data that the initial event would be a scattering complex $\left[\mathrm{e}^{-}-\mathrm{NB}\right]$ where the "continuum" excess electron would settle into a DBS anionic complex with the NB partner at its neutral geometry and that a further stabilization into a VBS anion of the NB species would occur via an intramolecular vibrational energy transfer process during the experimental observation.

Thus, the above studies indicate that internal restructuring of anionic species from DBS to VBS configurations (or to VBS-like excited states when the corresponding stable anions do not exist in isolated targets [17]), could occur first via a diffuse excess electron at near-zero energy with respect to the polar target. Their spatial shapes play an important role in the physics that leads to stable anions' final formation since the spatial proximity of such nearthreshold electrons increases overlap effects with the final states and favours anions' restructuring. They are further affected by environmental dynamics and by the operating conditions of the impinging electrons: from the solutestate of biosystems to the low-temperature, low-density 
characteristics of the interstellar space anions [14] the role of DBS states has been shown to provide pathways for structural rearrangements and energy redistributions after a molecule interacts with low-energy scattering electrons.

\section{The present scattering model}

In the following we shall describe the quantum dynamics of low-energy electron/positron scattering methods employed to generate $3 \mathrm{D}$ spatial wavefunctions (real part) of the excess lepton interacting with a "supercritical" polar NB target. The interaction forces, although dominated at low energies by the potential term of equation (1), need to be more realistically described by accurate representations of all its additional contributing terms (as reported below) to obtain a final picture for the scattered particle which is as accurate as possible for such large polyatomic targets. The method is quite general and has been employed by us over many years to describe the quantum dynamics of extra electrons/positrons interacting with polyatomic molecules (for a general review of the method see Refs. [33,34]).

\subsection{The SCE scattering equations}

In order to obtain the scattering cross sections for polyatomic molecules, we need to solve the Schroedinger equation for the total system

$$
(H-E) \Psi=0
$$

at the total energy $E$, for the corresponding wavefunction $\Psi$. Here $H$ is the total Hamiltonian given by:

$$
\hat{H}=\hat{H}_{m o l}+\hat{K}+\hat{V}
$$

where $\hat{H}_{m o l}, \hat{K}$ and $\hat{V}$ represent the operators of the molecular Hamiltonian, kinetic energy of the scattered electron and the interaction between the incident electron and the target molecule, respectively. The $\hat{H}_{m o l}$ further consists, in general, of the rotational and vibrational parts

$$
\hat{H}_{m o l}=\hat{H}_{r o t}+\hat{H}_{v i b}
$$

whereby we exclude, at the collision energies considered, the electronic excitations, the Ps formation (in case of positron scattering) and the roto-vibrational excitation channels. The total wavefunction $\Psi$ described in the molecular frame (MF) reference system in which the $z$ axis is taken along the direction of the main molecular axis, is expanded around a single-center as

$$
\Psi\left(\mathbf{r}_{1} \cdots \mathbf{r}_{Z}, \mathbf{r}_{l p t} \mid \mathbf{R}\right)=\Psi_{m o l}\left(\mathbf{r}_{1} \cdots \mathbf{r}_{Z} \mid \mathbf{R}\right) \phi\left(\mathbf{r}_{l p t} \mid \mathbf{R}\right)
$$

where

$$
\phi\left(\mathbf{r}_{l p t} \mid \mathbf{R}\right)=\sum_{\pi \mu \ell h} r_{l p t}^{-1} u_{\ell h}^{\pi \mu}\left(r_{l p t} \mid \mathbf{R}\right) X_{\ell h}^{\pi \mu}\left(\hat{r}_{l p t}\right) .
$$

In equation (5) $\mathbf{r}_{i}$ represents the position vector of the $i$ th electron among the $Z$ bound electrons of the target, taken from the center of mass. The quantity $\Psi_{m o l}$ represents the electronic wavefunction for the molecular target at the nuclear geometry $R$. The continuum function $\phi\left(\mathbf{r}_{l p t} \mid R\right)$ refers to the wavefunction of the scattered lepton (lpt) (i.e. either a continuum electron or a continuum positron as projectiles) under the full action of the field created by the molecular electrons and their response to the impinging lepton as described below. Each $u_{\ell h}^{\pi \mu}$ is the radial part of the wavefunction for the incident particle and the $X_{\ell h}^{\pi \mu}$ are the symmetry-adapted angular basis functions discussed earlier [33] which we will not repeat further here. The label $\pi$ stands for the irreducible representation (IR), $\mu$ distinguishes the components of the basis for each IR, respectively. Since the molecular rotations and vibrations are often slow when compared with the velocity of the impinging electrons considered in the present study, we may apply the fixed-nuclei approximation (FN, [33]) that ignores the molecular term of $\hat{H}_{m o l}$ in equation (3) and fixes the values of all $\mathbf{R}$ at their equilibrium locations in each of the target molecules. We then solve the Schroedinger equation in the FN approximation, make use of the MF system rather than the space-frame (SF) reference system: the two systems are related through a frame transformation scheme given, for example, by $[34,35]$.

After substituting equation (5) into (2) under the FN approximation, we obtain a set of coupled differential equations for $u_{\ell v}$, where, for simplicity, $v$ represents $(\pi \mu h)$ collectively:

$$
\begin{aligned}
\left(\frac{d^{2}}{d r^{2}}-\frac{\ell(\ell+1)}{r_{l p t}^{2}}+k^{2}\right) & u_{\ell v}\left(r_{l p t} \mid \mathbf{R}\right) \\
& =2 \sum_{\ell^{\prime} v^{\prime}}\left\langle\ell v|\mathbf{V}| \ell^{\prime} v^{\prime}\right\rangle u_{\ell^{\prime} v^{\prime}}\left(r_{l p t} \mid \mathbf{R}\right)
\end{aligned}
$$

with

$$
\left\langle\ell v|\mathbf{V}| \ell^{\prime} v^{\prime}\right\rangle=\int d \hat{r}_{l p t} X_{\ell v}\left(r_{l p t}\right) V\left(r_{l p t} \mid \mathbf{R}\right) X_{\ell^{\prime} v^{\prime}}\left(r_{l p t}\right) .
$$

Solving equation (7) under the boundary conditions that the asymptotic form of $u_{\ell v}$ is represented by a sum containing the incident plane wave of the projectile and the outgoing spherical wave, we obtain the corresponding S-matrix elements $S_{\ell v, \ell^{\prime} v^{\prime}}$. The actual numerical procedure we have employed to solve that equation was given in detail in references [36,37]. After transforming the MF quantities into the SF frame, the integral cross section (ICS) for the elastic scattering, rotationally summed over molecular rotations, is given by:

$$
Q=\frac{\pi}{k^{2}} \sum_{\ell v} \sum_{\ell^{\prime} v^{\prime}}\left|T_{\ell v, \ell^{\prime} v^{\prime}}\right|^{2}
$$

where $T_{\ell v, \ell^{\prime} v^{\prime}}=\delta_{\ell \ell^{\prime}, v v^{\prime}}-S_{\ell v, \ell^{\prime} v^{\prime}}$.

\subsection{Modeling the interaction forces}

For a target which has a closed shell electronic structure, with $n_{o c c}=N / 2$ doubly occupied molecular orbitals, 
the static-exchange (SE) potential has the following form, in the case of an impinging electron:

$$
\begin{aligned}
V_{S E} & =\sum_{\gamma=1}^{M} \frac{Z_{\gamma}}{\left|\mathbf{r}-\mathbf{R}_{\gamma}\right|}+\sum_{i=1}^{n_{o c c}}\left(2 \hat{J}_{i}-\hat{K}_{i}\right) \\
& =V_{s t}-\sum_{i=1}^{n_{o c c}} \hat{K}_{i}
\end{aligned}
$$

where $\hat{J}_{i}$ and $\hat{K}_{i}$ are the usual static potential and the nonlocal exchange potential operator, respectively. We further model the correlation and polarization effects via the following optical potential:

$$
V^{c p}(\mathbf{r})= \begin{cases}V^{\text {corr }}(\mathbf{r}), & \text { for } \mathbf{r}<\mathbf{r}_{\text {match }} \\ V^{\text {pol }}(\mathbf{r}), & \text { for } \mathbf{r}>\mathbf{r}_{\text {match }}\end{cases}
$$

employing density related models which have been described before for the short-range correlation effects [36]. We then generate the exchange interaction with the Free-Electron-Gas-Exchange model proposed by Hara (HFEGE, [38]), VHFEGE:

$$
V_{H F E G E}(\mathbf{r} \mid \mathbf{R})=-\frac{2}{\pi} K_{F}(\mathbf{r} \mid \mathbf{R})\left[\frac{1}{2}+\frac{1-\eta^{2}}{4 \eta} \ln \left(\frac{1+\eta}{1-\eta}\right)\right] .
$$

When dealing with closed-shell systems as the present case, the electronic density $\rho(\mathbf{r} \mid \mathbf{R})$ is given as:

$$
\begin{aligned}
\rho(\mathbf{r} \mid \mathbf{R})= & \int \mid \operatorname{det}\left\|\phi_{1}\left(\mathbf{r}_{1}\right) \phi_{2}\left(\mathbf{r}_{2}\right) \ldots \phi_{n_{e}}\left(\mathbf{x}_{n_{e}}\right)\right\|^{3} \\
& \times d \mathbf{x}_{1} d \mathbf{x}_{2} \ldots d \mathbf{x}_{n_{e}}
\end{aligned}
$$

is evaluated by assigning 2 as the occupation number of each of doubly occupied MOs in the neutral targets of the present study. In other words, the scattered electron initially interacts with the neutral molecular targets at the fixed geometry of their equilibrium structures. This final potential provides the so-called static-modelexchange-correlation-polarization (SMECP) potential accounting for the interaction forces between the impinging free electron and the target molecule.

The coupled set of integrodifferential equations now take the form:

$$
\begin{aligned}
& {\left[\frac{d^{2}}{d^{2} r}-\frac{\ell(\ell+1)}{r^{2}}+2(E-\epsilon)\right] f_{\ell h}^{\pi \mu}(\mathbf{r} \mid \mathbf{R})} \\
& =2 \sum_{\ell^{\prime} h^{\prime}} V_{\ell h, \ell^{\prime} h^{\prime}}^{\pi \mu}(r) f_{\ell h}^{\pi \mu}(r),
\end{aligned}
$$

indent where the potential coupling elements are

$$
\begin{aligned}
V_{\ell h, \ell^{\prime} h^{\prime}}^{\pi \mu} & =\left\langle X_{\ell^{\prime} h^{\prime}}^{\pi \mu}(\hat{r})|V(\mathbf{r})| X_{\ell h}^{\pi \mu}\right\rangle \\
& =\int d \hat{r} X_{\ell^{\prime} h^{\prime}}^{\pi \mu} V(\mathbf{r}) X_{\ell h}^{\pi \mu}
\end{aligned}
$$

The numerical solutions of the coupled equations produce the relevant K-matrix elements which will in turn yield the final integral cross sections [37].
In order to further test the modeling of density-related exchange and correlation effects described above, in the present multichannel quantum scattering calculations we have also employed different models for the treatment of the continuum-bound electron-electron dynamical correlation effects:

(a) orthogonality constraints. Since the scattering electron wavefunction is not describing exactly a virtual state from the target DFT molecular orbitals (given the marked effects caused by the added scattering correlation-polarization parts of the interaction) it is not anymore an eigenfunction of the hamiltonian for the target molecular electrons. Hence, one must make sure that it remains orthogonal to the bound orbitals of the same symmetry [33]. For polyatomic targets such constraint is usually satisfied since the static and exchange contributions dominate. However, when enforcing this orthogonality, one makes sure that the nodal structure of the scattered electron remains the correct one also in the outer regions of the target density and therefore allows for an increase of overall attractive effects from the target nuclei [34];

(b) using a different short-range correlation potential. In the treatment reported before we have employed the form described in reference [33] and obtained earlier by Perdew and Zunger PZ, [39,40]. A more attractive density functional form for the short-range correlation had been suggested by Padial and Norcross (PN, [41]) for diatomic targets and further implemented by us for polyatomic molecules [42]. It yielded interesting results in terms of good agreement with existing experiments (for a recent comparison see Ref. [43]). We further implemented a more computationally expensive alternative to evaluate exchange, one based on the Schwinger variational expression of the scattering matrix [44] that further included Pade' approximants corrections. Briefly, the interative treatment of the exchange interaction consists in dividing the interaction into two parts: an approximate, local potential $V_{L}$ that includes an initially simplified, semiclassical exchange form (SME, [36]), plus a difference potential $V_{D}$ which adds the correction due to using exact exchange interaction. The latter is initially defined as $V_{H F E G E}-V_{S M E}$ to start the interactive process. The solution to a purely local problem via the potential $V_{T}=V_{L}+V_{D}$ could be written as:

$$
H_{L} \Phi_{L}=E \Phi_{L}
$$

where $H_{L}=-1 / 2 \nabla^{2}+V_{L}$ and the associated Green's function is defined as:

$$
\left(E-H_{L}\right) G_{L}=1 .
$$

Any element of the final scattering matrix $K$ for the full potential $V_{T}$ can be written as:

$$
K_{p q}^{T}=K_{p q}^{L}+K_{p q}^{D}
$$

where the added correction is:

$$
K_{p q}^{D}=-2\left\langle\Phi_{L}\left|V_{D}\right| \Phi_{L}\right\rangle
$$


which can also be written via a Born series expansion [44]

$$
K_{p q}^{D}=-2 \sum_{j=0}^{\infty}\left\langle\Phi_{p}\left|V_{D}\left(G_{L} V_{D}\right)^{j}\right| \Phi_{q}\right\rangle .
$$

The convergence of this sum turns out to be greatly enhanced by using Pade' approximants of $[\mathrm{N} / \mathrm{N}]$ order, thereby allowing the calculations to incrementally modify the potential by using increasingly more accurate forms of the Green's function $G_{L}$ in equation $(20)[43,44]$. Since the corresponding modeling of the interaction forces in the case of an impinging, low-energy positron have been described before many times (e.g. see Refs. [45-47]), we shall not be repeating them here again since we shall focus the present study on the attachment of extra electrons. The case of the positrons will only be mentioned for a brief comparison.

\subsection{Present calculations on adenine and guanine}

The examples below shall correspond to results obtained at a collision energy of $1 \mathrm{meV}$, unless otherwise stated. This value was chosen since it represents a low enough collision energy that one can consider the scattering via a complicated, multi-centered interaction potential for the excess electron very close to the energy threshold values similar to those surmised by experiments [18]. The singlecenter expansion of all the terms has been extended to $L_{\max }=23$, which corresponds to 2116 partial waves describing the scattering event. Numerical convergence was thus obtained within a few percent for all numerical observables. The SCE description of all the many bound molecular orbitals (MOs) occupied in each target molecule yielded a normalization value well above 0.9 for all of them, which is accurate enough for the present problem since it allows a numerically faithful representation of all the MO-derived interaction terms described in the previous subsections. We further generated the Diabatic Representation of the interaction potential matrix, as described in [33], and employed in the generation of the spatial scattering functions 256 diabatic partialwaves within a 3D box of $100 \AA$. All scattering results were found to be numerically converged even when using smaller boxes, thereby confirming the quality of the final calculations over a larger scattering grid. The data in Figure 1 report the molecular geometries we have employed in our present study, the orientation of the dipole components and the partial Mulliken's charges on each of the atoms.

It is interesting to note from the data in that figure that both species have permanent dipole moments which are well above the threshold value (either 1.67 or $2.5 \mathrm{D}$ ). One should also keep in mind, however, that the actual description of all the important contributions to the interaction of the purinic bases with an external electron is clearly more complicated: the final regions of the target molecules where the excess electron will be collocated will not depend only on the sign and orientations of the

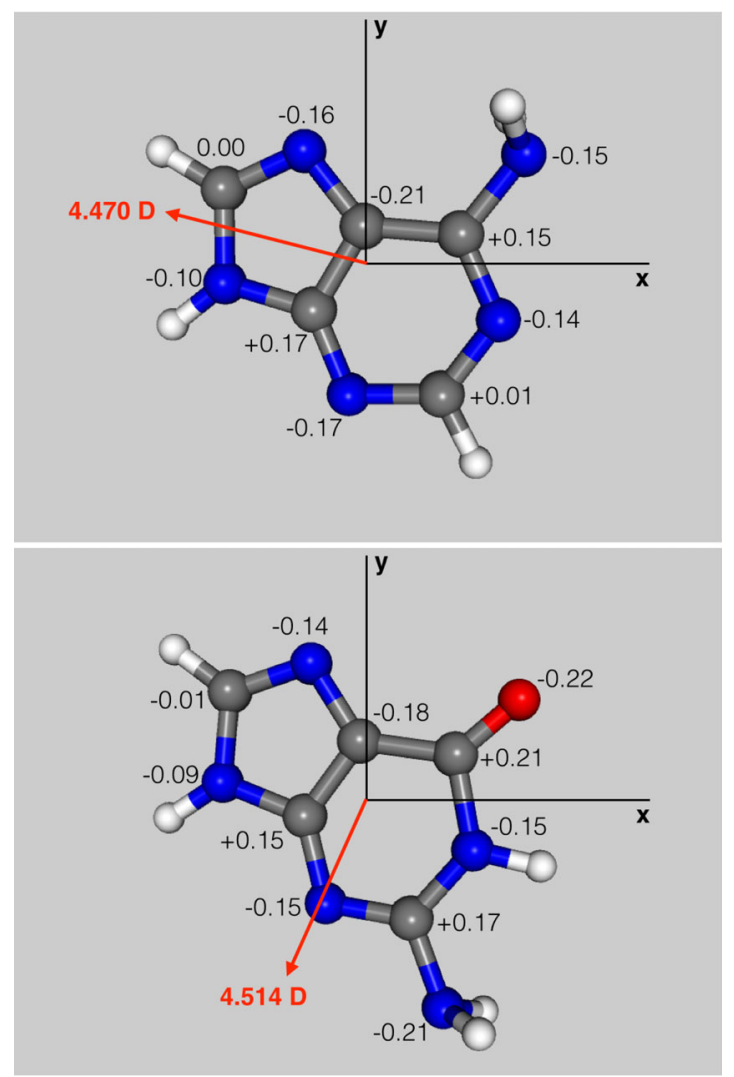

Fig. 1. Computed structures of the two purinic DNA bases. From top to bottom: adenine and guanine. The orange solid arrows show their molecular dipole moments in the $x y$ plane. The Mulliken partial charges are also reported for all atoms but hydrogens. See main text for further details.

supercritical dipoles but also from the higher multipolar terms and from the bound electrons' charge distributions. The first step in the analysis of the scattering events near threshold energies therefore requires to know the overall shape of the multipolar coefficients associated with the strongly anisotropic interactions between the fixedgeometry molecular target's nuclei, its large number of bound electrons and the low-energy impinging electron. The data reported by Figures 2 and 3 show the lowest components of the large sum over multipolar coefficients discussed in the previous section and employed in the actual calculations. Given the very low collision energy of the incoming electrons, we report there only the $\ell=0$ ( $s$-wave) and the $\ell=1$ ( $p$-wave) components of the full interactions. Figure 2 deals with the adenine target molecule, while Figure 3 presents results for the guanine. To our knowledge, no previous spatial analysis of the near-threshold scattering states for the present NBs has been found in the relevant literature

The curves reported in the two figures are all measured from the molecular center of mass, where the permanent dipole is also centered, and clearly show for both molecular targets that the spherical interaction associated to the $s$-wave behaviour is always strongly attractive in all directions of approach, as is naturally expected to occur from the polarization-correlation forces acting close 


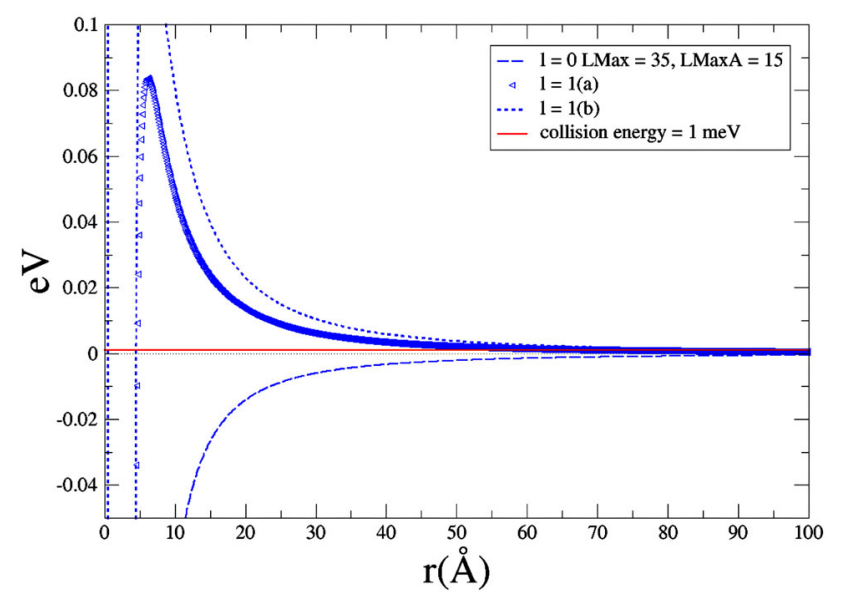

Fig. 2. Lowest- $\ell$ contributing terms, $V_{\ell m}^{t o t}\left(r_{l p t}\right)$ of the interaction between electrons and adenine. See main text for further details.

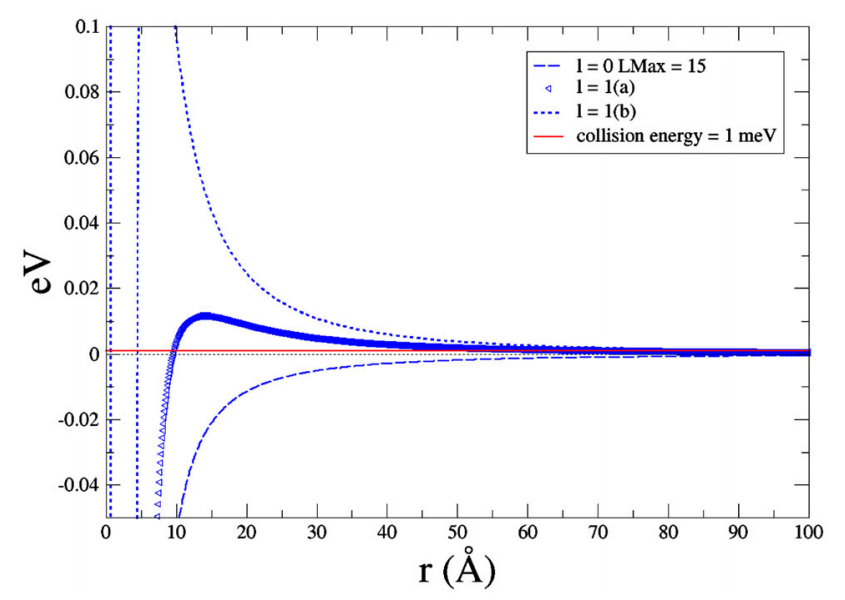

Fig. 3. Lowest- $\ell$ contributing terms, $V_{\ell m}^{\text {tot }}\left(r_{l p t}\right)$ of the interaction between electrons and guanine. See main text for further details.

to the molecular "volume". On the other hand, the two components for the $\ell=1$ ( $p$-wave) interaction, the one which is strongly orientational and is chiefly driven by the dipolar potential at long-range, exhibit the presence of a marked barrier in both components. Given the low collision energy of the threshold electrons we are considering in this analysis, it follows that such interactions are already keeping the impinging electrons quite far outside the molecular space. If we were to also represent the next term in the multipolar interaction, i.e. the $\ell=2$ ( $d$-wave) component of the potentials, such terms will be even more strongly repulsive and therefore would play a marginal role during the scattering process and in shaping the full scattering wavefunctions. Thus, we can say that the approaching excess electrons at threshold will essentially be driven by the $\ell=0$ and the $\ell=1$ components and will chiefly behave like an $(s+p)$-wave during the initial scattering event.

In comparing the behavior of the $p$-wave potential contributions in the two systems, it is also significant to note that the repulsive barrier has a higher and more closely

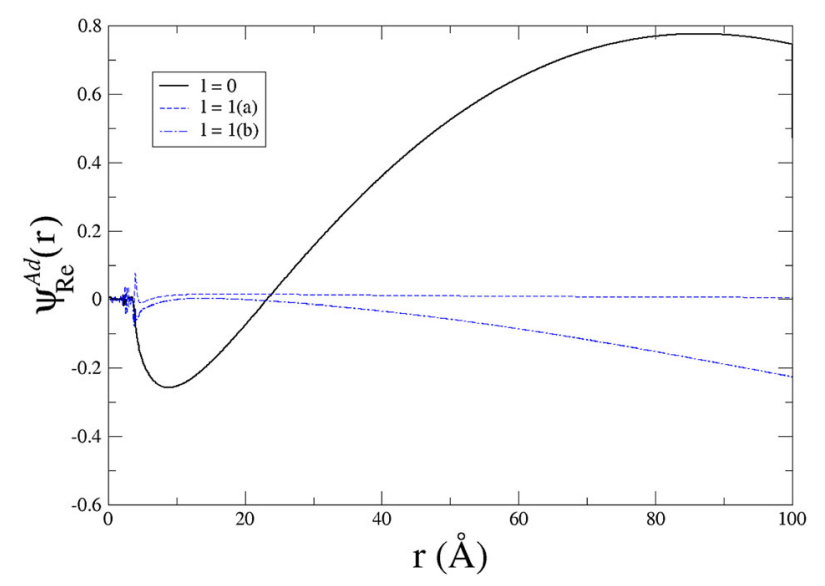

Fig. 4. Lowest- $\ell$ contributing terms for the real parts of the scattering electron's wavefunctions off the adenine molecular target. See main text for further details.

positioned maximum for adenine than in the case of guanine: a qualitative explanation comes from the much larger number of bound electrons in the case of the latter as opposed to the former target. Hence, their role of screening the repulsive nuclear charges in the regions closer to the center-of-mass will be more effective in guanine and will produce a final potential that is less repulsive than the one for adenine. The near-threshold electron is therefore expected to come closer when impinging on the former molecule than when interacts with the latter. Such differences will also be further analysed below.

\section{The adenine and guanine DSS molecular anions}

An interesting series of properties of the excess electrons at threshold energies could be obtained by first observing the behaviour of the radial components (real part) of the scattering wavefunctions for the lowest contributing partialwaves. Figure 4 deals with the adenine wavefunction while Figure 5 presents the guanine's one.

In the case of adenine the inner region is more compact than in the case of guanine, where the othogonality node is located further out. In both cases, however, it is clear that the largest portions of the $s$-wave components are located way outside the molecular "volumes" of the two targets. This behaviour is also evident from the two components of the radial $p$-waves, where we see the extensive nodal structures close to the molecular nuclei and fairly little tunneling penetration of the impinging electrons. The data in these figures are clearly pointing at an extra electron being largely located well outside the molecules, exactly where one also expects to find the spatial locations of the dipole-driven bound states for the same systems. This aspect will be further analysed below.

The data provided by the radial components of the threshold scattering wavefunctions can of course be combined with their symmetry-adapted angular components to generate the corresponding three-dimensional (3D) features of such scattering states with respect to the target 


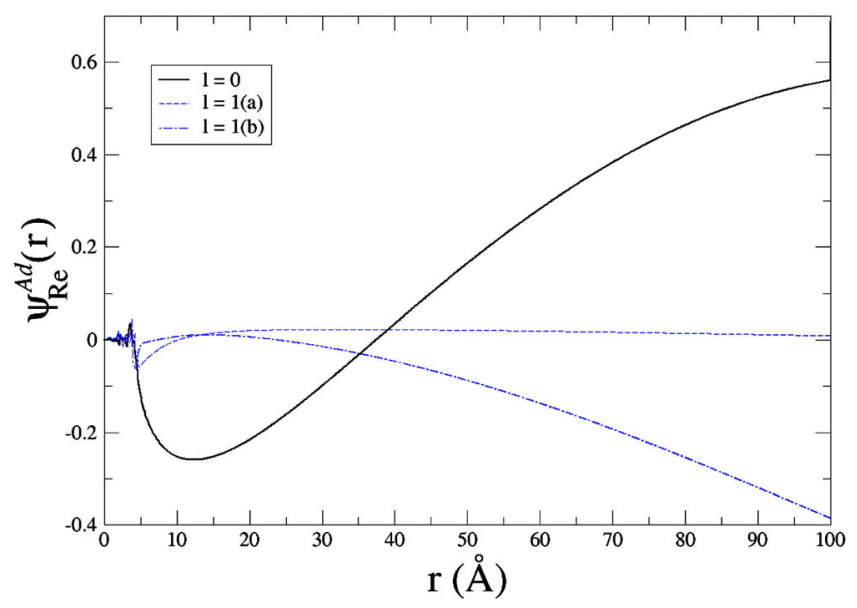

Fig. 5. Lowest- $\ell$ contributing terms for the real parts of the scattering electron's wavefunctions off the guanine molecular target. See main text for further details.

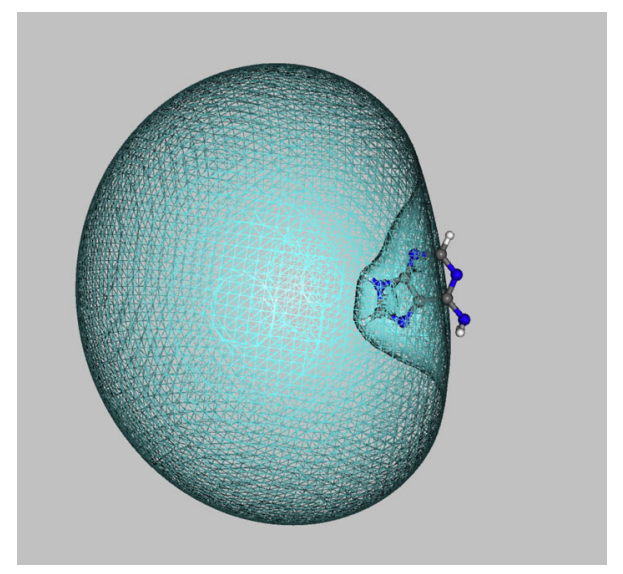

Fig. 6. Computed 3D maps of the near-threshold scattering wavefunction (real part) for the electron projectile off the adenine molecule using the value +0.003 for the cutoff. See text for further details.

molecules. In depicting such states, to emphasize their relative positioning, we shall assume that the molecules will not rotate during the time that the impinging electrons will be close to the molecules so that their fixed dipoles will control their short-range collocations. Figures 6 and 7 , respectively show the general shapes of the spatial wavefunctions. In the two figures we report the behaviour of the electron projectile and also that of its leptonic counterpart, the positron. In the case of the latter projectile, shown here to underline its complementary behaviour, the calculations have followed the details of the method employed by us in our earlier work references [45-47] and described there in much greater detail.

The data in Figure 6 show the spatially extended wavefunction of the DSS anion: we report the relative position of the adenine molecule with respect to the threshold electron. By considering the dipole's orientation of Figure 1, the diffuse electron is positioned at the outer end of the positive direction of the dipole, distributed largely on that

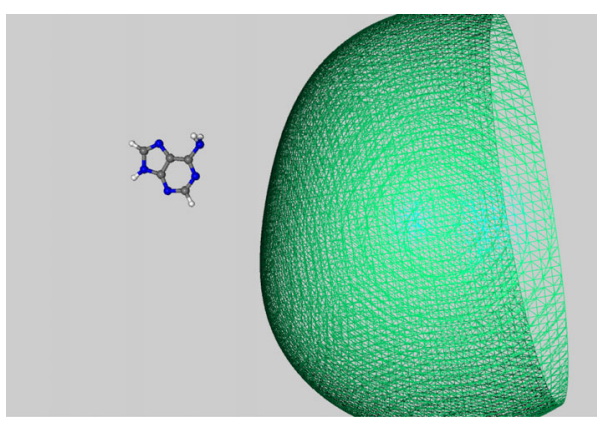

Fig. 7. Computed 3D maps of the near-threshold scattering wavefunction (real part) for the leptonic antiparticle, the positron, off the adenine molecule, using the value +0.003 for the cutoff. See main text for further details.

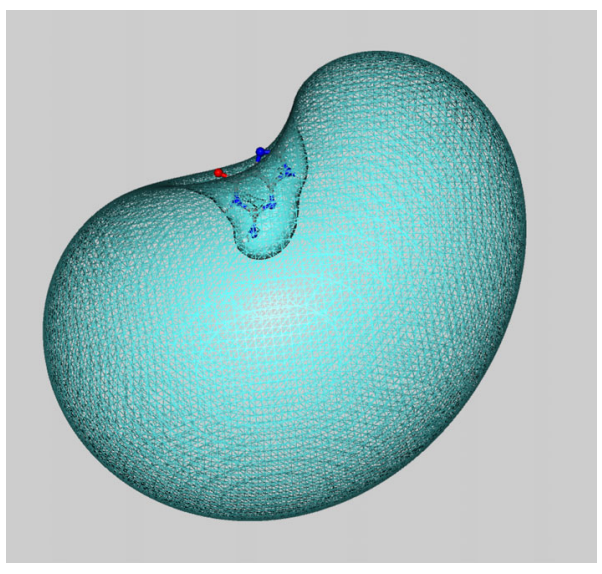

Fig. 8. Computed 3D maps of the near-threshold scattering wavefunction (real part) for the electron projectile off the guanine molecule using the value +0.003 for the cutoff. See main text for further details.

region of the molecular nuclei which are enveloped by the extra electron wavefunction.

On the other hand, when we now look at the 3D wavefunction for the corresponding antiparticle in Figure 7, we clearly see the opposite collocation of the threshold positron which maps the external molecular region but at the negative end of its permanent dipole. We further notice how its overall behaviour is that coming from a largely repulsive scattering potential: the correponding wavefunction is, on the same scale, much more diffuse and further outside with respect to that of the scattered electron.

The scattered wavefunction off the guanine molecule is presented by the data reported in Figures 8 and 9 for the same leptonic partners.

The orientation of the permanent dipole in this molecule (e.g. see Fig. 1) indicates that the diffuse, external lobe of the near-threshold electron interacting with the guanine target is indeed localized on the positive end of that dipole. We therefore see here the same behaviour already observed in Figure 6 and also extensively discussed in our earlier work (see Refs. $[45,46]$ ): the DSS anions correspond to near-zero-energy electrons in the continuum which get located on that area of the molecular space 


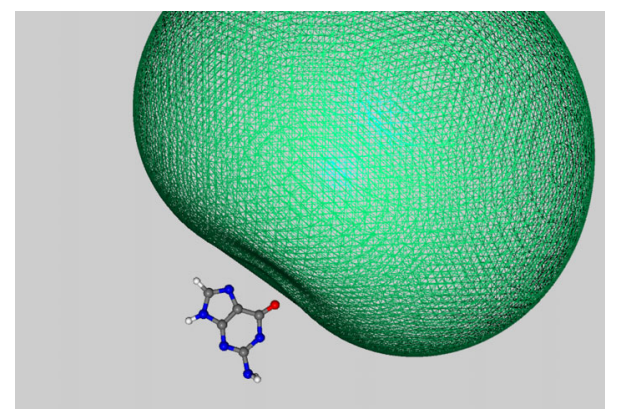

Fig. 9. Computed 3D maps of the near-threshold scattering wavefunction (real part) for the positron projectile off the guanine molecule using the value +0.003 for the cutoff. See main text for further details.

where the positive end of the permanent dipole is also pointing at.

The lower panel of Figure 9 reports the same type of 3D map corresponding now to the electron's antiparticle: the positron. What we see in that figure is that the electron's antiparticle is spatially more diffuse than the electron's wavefunction and that it locates itself on the negative ending of the molecular dipole moment, as observed for the adenine target in Figures 6 and 7 .

It is also interesting to note that the DNA nucleic acid bases exhibit a large variety of tautomeric configurations that show different response to electron attachment stabilization, a feature already extensively analysed in the current literature both experimentally and computationally (e.g. see Refs. $[27,29])$. Thus, that work has shown that adenine and guanine, which have a much smaller gas-phase affinity to an impinging excess electron than the pyrimidinic bases [48], also exhibit very different properties when less common tautomeric forms are considered [28]: the occurrence of enamine-imine transformations, for example, causes the formation of much more strongly bound VB states, the latter being even more likely to form in solutions after an $\mathrm{H}$ atom migration to a carbon atom [48-50]. Computational studies on the most stable structures of adenine in relation to the presence of the more weakly bound DB states have indicated that their binding energy is very close to zero and that it can become more stable in solutions through the orientational action of the solvent water molecules $[49,50]$. Furthermore, the computational studies related to such possible anions indicate them to be described by diffuse orbitals located outside the molecular structures, just as we found here to be the case for the near-threshold DSS scattering electrons for the same NB systems [51]. The fact that such scattering states would be located in similar regions of the molecular space as the weakly bound DBS anions is therefore an interesting result which provides a direct mechanism to stabilize anionic complexes formed in the energy continuum into bound anionic structures located within the same molecular regions. The comparison we report in Figure 10 for the adenine molecule provides computational evidence for such strong similarity of shapes.
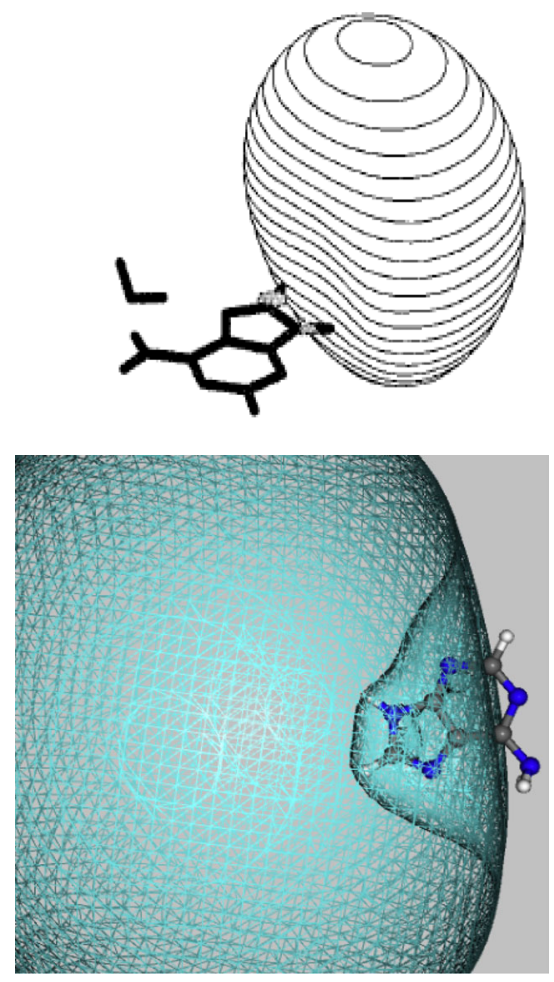

Fig. 10. Computed structures of the two excess electron's wavefuntions associated with the adenine molecule. from top to bottom: the shape of the electron's DBS state from reference [50], the spatial location of the DSS state for the same molecule. See main text for further details.

\section{Concluding remarks}

One of the common properties within the series of the DNA's NBs is the presence of fairly large values of permanent dipole moments. The chemical and physical consequences of this common property have been extensively studied over the years, especially in relation to their interactions with external electrons coming from their cell environments after ionization of the water molecules in the solvents (e.g. see Refs. [11,13]). In experiments and calculations, many of which have been dicussed in our Introduction, has been pointed out that such permanent dipoles play a significant role on the formation of bound anionic states, either in the gas phase or in a solvent environment. For systems like adenine and guanine, which were also found to have EA values close to zero, it was also discussed in both experiments and calculations that the presence of a large number of tautomeric configurations could lead to small structure modifications (e.g. induced by a solvent) which allow the formation of bound anions of the dipole-driven variety (DBS anions). Such 'intermediate' bound states could further turn into more tightly bound, and spatially more compact, VB anionic states [51,52] by additional, stronger perturbations from their environments. The discussion reported in the present work indicates that these near-threshold scattering states can be accessed by the excess electrons coming from the chemical environment of the DNA nucleic acid bases. 
The latter zero-energy electrons can thus interact with the NB targets chiefly via their supercritical, permanent dipole moment and their corresponding states turn out to be very similar (in terms of their spatial extension and relative collocation within the molecular space) to those found by calculations to be weakly bound as DBS anions: such DB anionic complexes provide important intermediates toward the final formation of $\mathrm{VB}$ anions in the cell environments $[52,53]$.

In the parallel language of the radiative stabilization mechanisms under the conditions of the Interstellar Medium [54,55], one can then say that the overlap integrals between DSS and DBS configurations of a given molecular environment are now expected to be very large and therefore favour radiative transitions between such states. In the more dense conditions of a biological environment this concept translates into the suggestion that the small energy gaps between DSS and DBS anions could favour the formation of the DBS structures of the NB molecules.

One can therefore argue that the free electrons in the biological environments of the NB targets $[52,53]$ can follow alternative paths to those provided by dissociative electron attachment (DEA) processes and form, through a more gentle mechanism, anionic species where the whole of the molecule is preserved. This could then be qualitatively described as follows: $\mathrm{M}+\mathrm{e}^{-}=>[\mathrm{DSS}]=>[\mathrm{DBS}]=>$ $\mathrm{M}^{-}$. As mentioned before, the recent experimental studies on the iodide-adenine binary complexes [18] indeed suggest that specific tautomeric forms of the adenine partner within the complexes can make that systems follow the formation of adenine's weakly-bound anions before observing the detaching electron after the complex's breakup. To reach the intermediate DB state of such molecular anions therefore requires the occurrence, above the energy threshold, of low-energy scattering states which are also driven by the supercritical permanent dipoles and that strongly resemble, in shape and spatial collocation, the weakly bound DB anionic configurations. The final molecular anion can thus be reached via the former complex with very little energy being spent for the rearrangement process. Although the above analysis is still at the conjectural level, the present computational verification of the spatial similarities between DSS and DBS anions in NBs, linked to the evidence from the experimental analysis [18] of the occurrence of such transitions, indicate the above path to be a realistic possibility.

The support of the supercomputing center of the University of Innsbruck is gratefully acknowledged. F.A.G. gratefully thanks the extensive correspondence and the the helpful information provided by Prof. L. Adamovictz and Prof. Daniel Neumark during the preparation of the present work. F.C. acknowledges support from the Austrian FWF Research Agency while this work was completed.

\section{References}

1. E. Fermi, E. Teller, Phys. Rev. 72, 399 (1947)

2. A.S. Wightman, Phys. Rev. 77, 521 (1949)
3. M.H. Mittelman, V.P. Myerscough, Phys. Rev. 23, 545 (1966)

4. J.-M. Levy-Leblond, Phys. Rev. 153, 1 (1967)

5. K. Connolly, D.J. Griffith, Am. J. Phys. 75, 524 (2007)

6. W.B. Brown, R.E. Roberts, J. Chem. Phys. 46, 2006 (1967)

7. O.H. Crowford, W.R. Garrett, J. Chem. Phys. 66, 4968 (1977)

8. J. Simons, Acc. Chem. Res. 39, 772 (2006)

9. H. Hotop, M.-H. Ruf, M. Allan, I.I. Fabrikant, Adv. At. Mol. Phys. 49, 85 (2003)

10. E. Alizadeh, L. Sanche, Chem. Rev. 112, 5578 (2012)

11. N.A. Oyler, L. Adamowiz, J. Phys. Chem. 97, 11122 (1993)

12. C. Desfrancois, H. Abdoul-Carima, N. Khelifa, J.P. Schermann, Phys. Rev. Lett. 73, 2436 (1994)

13. J. Simons, J. Phys. Chem. A 112, 6404 (2008)

14. F. Carelli, T. Grassi, F.A. Gianturco, A\&A 428, 1181 (2013)

15. K. Koyanagi, Y. Kita, Y. Shigata, M. Tachikawa, ChemPhysChem 15, 16208 (2013)

16. X. Li, M.D. Sevilla, Adv. Quantum Chem. 52, 59 (2007)

17. C. Desfrancois, V. Periquet, Y. Bouteiller, J.P. Schermann, J. Phys. Chem. A 102, 1274 (1998)

18. A.B. Stephansen, S.B. King, Y. Yokoi, Y. Minoshima, W.-L. Li, A. Kunin, T. Takayanagi, D.M. Neumark, J. Chem. Phys. 143, 104308 (2015)

19. I. Baccarelli, I. Bald, F.A. Gianturco, E. Illenberger, J. Kopyra, Phys. Rep. 508, 1 (2011)

20. J.A. Stockdale, F.J. Davis, R.N. Compton, C.E. Klots, J. Chem. Phys. 60, 4279 (1974)

21. R. Hashemi, E. Illenberger, J. Phys. Chem. 95, 6402 (1991)

22. H. Haberland, C. Ludewigt, H.G. Schindler, D.R. Warsuop, J. Chem. Phys. 81, 3742 (1984)

23. K.R. Lykke, R.D. Mead, W.C. Lineberger, Phys. Rev. Lett. 52, 2221 (1984)

24. C. Desfrancois, H. Abdoul-Carime, C. Adjami, N. Khelifa, J.P. Schermann, Europhys. Lett. 26, 25 (1994)

25. F. Lecomte, S. Carles, C. Desfrançois, M.A. Johnson, J. Chem. Phys. 113, 10973 (2000)

26. L. Suess, Y. Liu, R. Parthasarathy, F.B. Dunning, J. Chem. Phys. 119, 12890 (2003)

27. M. Cannon, Y. Liu, L. Suess, F.B. Dunning, J. Chem. Phys. 128, 244307 (2008)

28. R.A. Bachorz, W. Klopper, M. Gutowski, X. Li, K.H. Bowen, J. Chem. Phys. 129, 054309 (2008)

29. M. Haranczyk, M. Gutowski, X. Li, K.H. Bowen, Proc. Natl. Acad. Sci. 104, 4804 (2007)

30. A.F. Jalbout, L. Adamowicz, Adv. Quantum Chem. 52, 231 (2007)

31. S.B. King, M.A. Yendell, A.B. Stephansen, D.M. Neumark, J. Chem. Phys. 141, 224310 (2014)

32. M.A. Yandell, S.B. King, D.M. Neumark, J. Chem. Phys. 140, 184317 (2014)

33. F.A. Gianturco, A. Jain, Phys. Rep. 143, 347 (1986)

34. F.A. Gianturco, R.R. Lucchese, Rev. Phys. Chem. 15, 429 (1996)

35. E.S. Chang, U. Fano, Phys. Rev. 46, 173 (1972)

36. F.A. Gianturco, R. Curik, N. Sanna, J. Phys. B 33, 2705 (2000)

37. N. Sanna, F.A. Gianturco, R.R. Lucchese, J. Chem. Phys. 100, 6464 (1994)

38. S. Hara, J. Phys. Soc. Jpn 22, 710 (1967)

39. F.A. Gianturco, Physica Scripta T 23, 141 (1988) 
40. J.P. Perdew, A. Zunger, Phys. Rev. B 23, 5048 (1981)

41. N.T. Padial, D.W. Norcross, Phys. Rev. A 29, 1742 (1984)

42. F.A. Gianturco, A. Jain, L.C. Pantano, J. Phys. B 20, 571 (1987)

43. F.A. Gianturco, R.R. Lucchese, J. Chem. Phys. 108, 6144 (1998)

44. R.R. Lucchese, V. Mc Koy, Phys. Rev. A 28, 1382 (1983)

45. F. Carelli, J. Franz, F.A. Gianturco, Mol. Phys. 114, 213 (2016)

46. F. Carelli, F.A. Gianturco, Eur. Phys. J. D 67, 268 (2013)

47. J. Franz, F.A. Gianturco, Eur. Phys. J. D 68, 183 (2014)

48. S.S. Wesolowski, M.L. Leininger, P.N. Pentchew, H.F. Schaefer, III, J. Am. Chem. Soc. 123, 4023 (2001)

49. E.D. Raczinska, M. Makowski, K. Zyentara-Rytter, K. Kolczynska, T.M. Stepniewsli, M. Hallmann, J. Phys. Chem. A 117, 1371 (2013)

50. A.F. Jalbout, L. Adamowicz, J. Phys. Chem. A 105, 1033 (2001)
51. G.H. Roehrig, N.A. Oyler, L. Adamowicz, J. Phys. Chem. 99, 14285 (1995)

52. J.H. Hendricks, S.A. Lyapustina, H.L. De Clercq, J.T. Snodgrass, K.H. Bowen, J. Chem. Phys. 104, 7788 (1996)

53. V. Periquet, A. Moreau, S. Carles, J.P. Schermann, C. DesFrançois, J. Electron Spect. Relat. Phenom. 106, 141 (2000)

54. F. Carelli, T. Grassi, F.A. Gianturco, A\&A 549, 103 (2013)

55. M. Satta, F.A. Gianturco, F. Carelli, R. Wester, ApJ 799, $288(2015)$

Open Access This is an open access article distributed under the terms of the Creative Commons Attribution License (http://creativecommons.org/licenses/by/4.0), which permits unrestricted use, distribution, and reproduction in any medium, provided the original work is properly cited. 